\title{
Growth and characterization of calcium pyrophosphate dihydrate grown in silica gel
}

\author{
$M$ DEEPA, $K$ RAJENDRA BABU and V $K$ VAIDYAN \\ Department of Physics, University of Kerala, Kariavattom, Thiruvananthapuram 695581, \\ India \\ MS received 22 June 1993; revised 11 November 1993
}

\begin{abstract}
Calcium pyrophosphate dihydrate crystals, which are responsible for various arthritic diseases, were grown in sodium metasilicate gel. Variations in gel density, pH value and concentration of the reactants were found to have profound effect on the growth of the crystais. The grown crystals were characterized using IR, XRD, TGA and optical microscopy.
\end{abstract}

Keywords. Calcium pyrophosphate dihydrate; gel; arthritic diseases; deposition.

\section{Introduction}

The arthritic diseases, namely pseudogout, pyrophosphate arthropathy and calcium pyrophosphate crystal deposition disease, are caused by the deposition of insoluble monoclinic, orthorhombic and triclinic calcium pyrophosphate dihydrate crystals within the articular tissues. The connective tissue matrix, especially the hyaline cartilage, can be considered as a gel (Pritzker et al 1981) and the formation of calcium pyrophosphate dihydrate is regulated by the physical and chemical states of the gel. In the present work an attempt has been made to grow calcium pyrophosphate dihydrate crystals in silica gel. The nucleation, growth and a few physicochemical properties are reported.

\section{Experimental}

The technique consists of incorporating one reagent into the gelling mixture and later diffusing another reagent into the gel leading to high supersaturation to initiate nucleation and crystal growth. The gel method of crystal growth is often used because of its simplicity and the quality of the crystal produced. In the present work, silica gel prepared from an aqueous solution of sodium metasilicate was used as the growth medium. Sodium metasilicate was prepared with gel densities ranging from 1.02 to $1.05 \mathrm{~g} / \mathrm{cm}^{3}$. Pyrophosphoric acid was prepared with concentrations from 0.125 to $3 \mathrm{M}$. Gels were prepared by mixing appropriate quantities of sodium metasilicate and pyrophosphoric acid, adjusting the $\mathrm{pH}$ in the range 5.5-7. After the setting of the gel, the feed solution of calcium chloride was added. Calcium pyrophosphate forms according to the reaction

$$
\mathrm{H}_{4} \mathrm{P}_{2} \mathrm{O}_{7}+2 \mathrm{CaCl}_{2} \cdot 2 \mathrm{H}_{2} \mathrm{O} \rightarrow \mathrm{Ca}_{2} \mathrm{P}_{2} \mathrm{O}_{7} \cdot 2 \mathrm{H}_{2} \mathrm{O}+4 \mathrm{HCl} \text {. }
$$

Three different sets of experiments were conducted by varying the gel density, $\mathrm{pH}$ and concentration of the feed solution. The grown crystals were characterized using IR, XRD, TGA and optical microscopy. 


\section{Observations}

Three different sets of experiments were conducted. To investigate the effect of gel density on the growth of the crystals, gel densities from 1.02 to $1.05 \mathrm{~g} / \mathrm{cm}^{3}$ were used. At a density of $1.05 \mathrm{~g} / \mathrm{cm}^{3}$, spherulitic crystals of calcium pyrophosphate dihydrate started forming within two days. When the gel density was varied from 1.02 to $1.05 \mathrm{~g} / \mathrm{cm}^{3}$, nucleation time decreased while the number of crystals per unit volume increased. It was observed that gel density has no pronounced effect on the quality of the crystals. In the second set of experiments, in which $\mathrm{pH}$ was varied from 5.5 to 7 , good-quality crystals were obtained at a $\mathrm{pH}$ of 6.5 ; with increase in $\mathrm{pH}$, the nucleation time and number of crystals decreased. Various concentrations of the incorporated calcium chloride solution affected the crystal size as well as the number of nucleation centres. With decrease in molarity of feed solution in the range $3 \mathrm{M}$ to $0.125 \mathrm{M}$ the nucleation time increased while the number of crystals decreased. Further, a decrease in crystal size was also observed. Good-quality crystals were observed at a molarity of $1 \mathrm{M}$ calcium chloride. The growth was completed in a period of two weeks. The grown crystals were washed with distilled water, dried and characterized using IR in the range 400 to $4000 \mathrm{~cm}^{-1}$, XRD in the $2 \theta$ range 0 to $100^{\circ}$, TGA in the temperature range 60 to $780^{\circ} \mathrm{C}$, and optical microscopy. In order to explain the growth kinetics of the crystals, the length of the crystals are plotted against time of growth. Their relationship (Law and Faust 1972) can be expressed as

$$
Y=K t \text {, }
$$

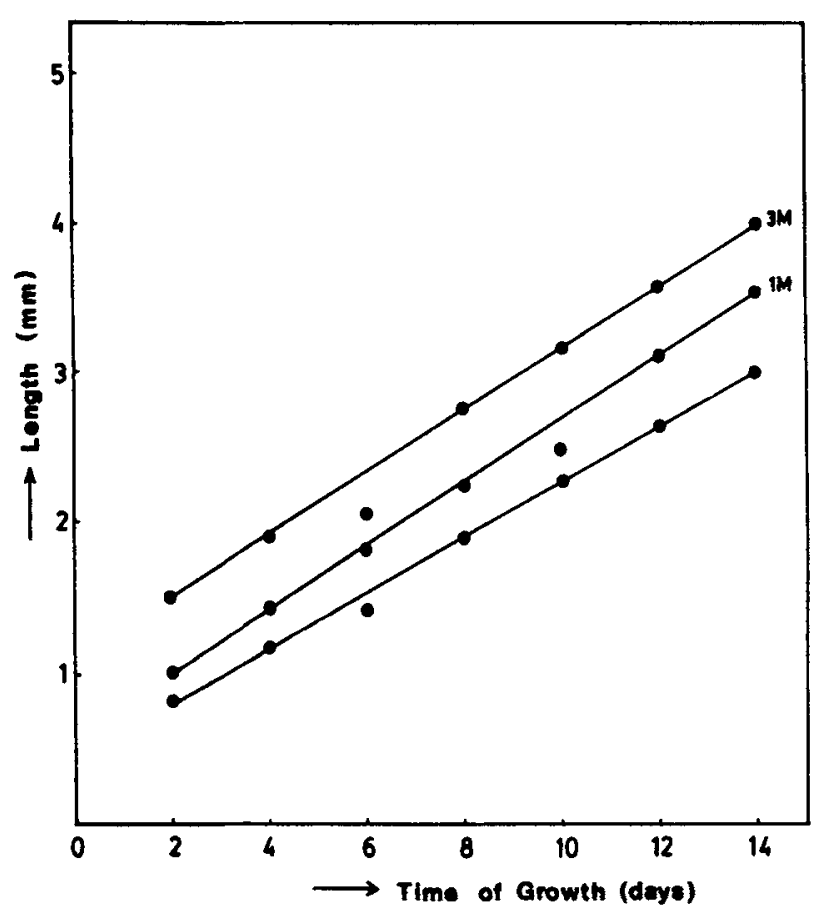

Figure 1. Plot of growth rate of the crystal with molarity of feed solution. 
where $Y$ is the length of the crystal, $t$ the time of growth, and $K$ the growth rate constant.

The plot of growth rate is given in figure 1. Figures 2, 3, 4 and 5 give the infrared spectrum, $X$-ray diffraction pattern, TG tracing and optical micrographs of the crystal respectively.

\section{Results and discussion}

\subsection{Infrared analysis}

The IR spectrum was recorded in the region 400 to $4000 \mathrm{~cm}^{-1}$. The spectrum (figure 2) shows bands similar to those reported in the literature (Corbridge and Lowe 1954). The bands observed between 3300 and $1640 \mathrm{~cm}^{-1}$ correspond to $\mathrm{O}-\mathrm{H}$ stretching and $\mathrm{O}-\mathrm{H}$ bending respectively. An imperfectly resolved doublet lies in the region $1170-1060 \mathrm{~cm}^{-1}$ corresponding to ionic $\mathrm{P}-\mathrm{O}$ stretching. At lower frequencies band seen at $1045-970 \mathrm{~cm}^{-1}$ can be assigned to other modes of $\mathrm{P}-\mathrm{O}$ stretching. Further one strong broad absorption band and weaker peaks seen between $940-870 \mathrm{~cm}^{-1}$ correspond to the harmonics of P-O-P stretching.

\section{$4.2 \quad X$-ray diffraction studies}

The X-ray diffraction powder pattern of the crystal recorded using $\mathrm{CoK}_{\alpha}$ source is given in figure 3 . The $d$ values calculated were found to be in close agreement with those obtained from ASTM card files and also from the literature (Brown 1963). The $d$ values obtained are $0.737,0.462,0.304 \mathrm{~nm}$, corresponding to $2 \theta$ values $10^{\circ}, 27.5^{\circ}$ and $33.5^{\circ}$ respectively. These $d$ values confirm the monoclinic nature of the crystal.

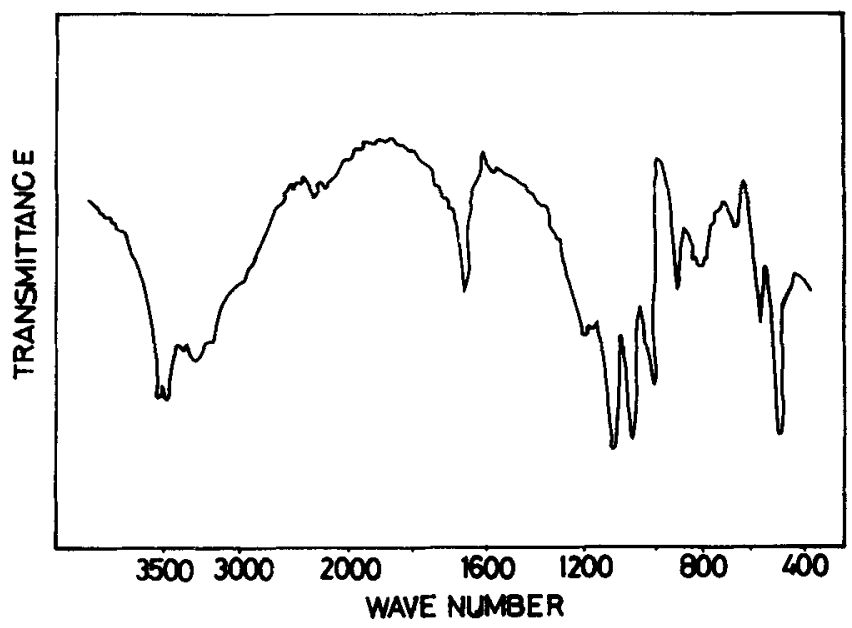

Figure 2. Infrared spectrum of gel-grown calcium pyrophosphate dihydrate in the region 400 to $4000 \mathrm{~cm}^{-1}$. 


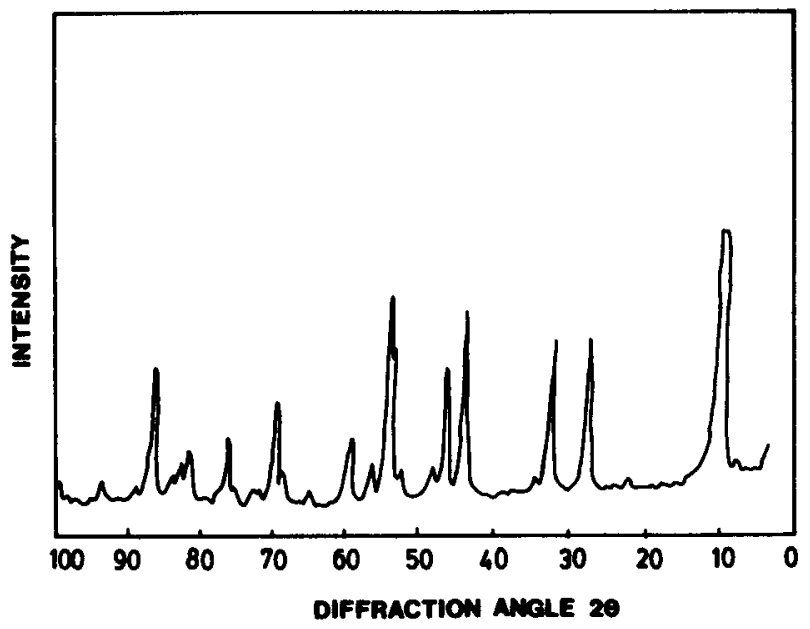

Figure 3. XRD scan of calcium pyrophosphate dihydrate in the $2 \theta$ range $0^{\circ}$ to $100^{\circ}$.

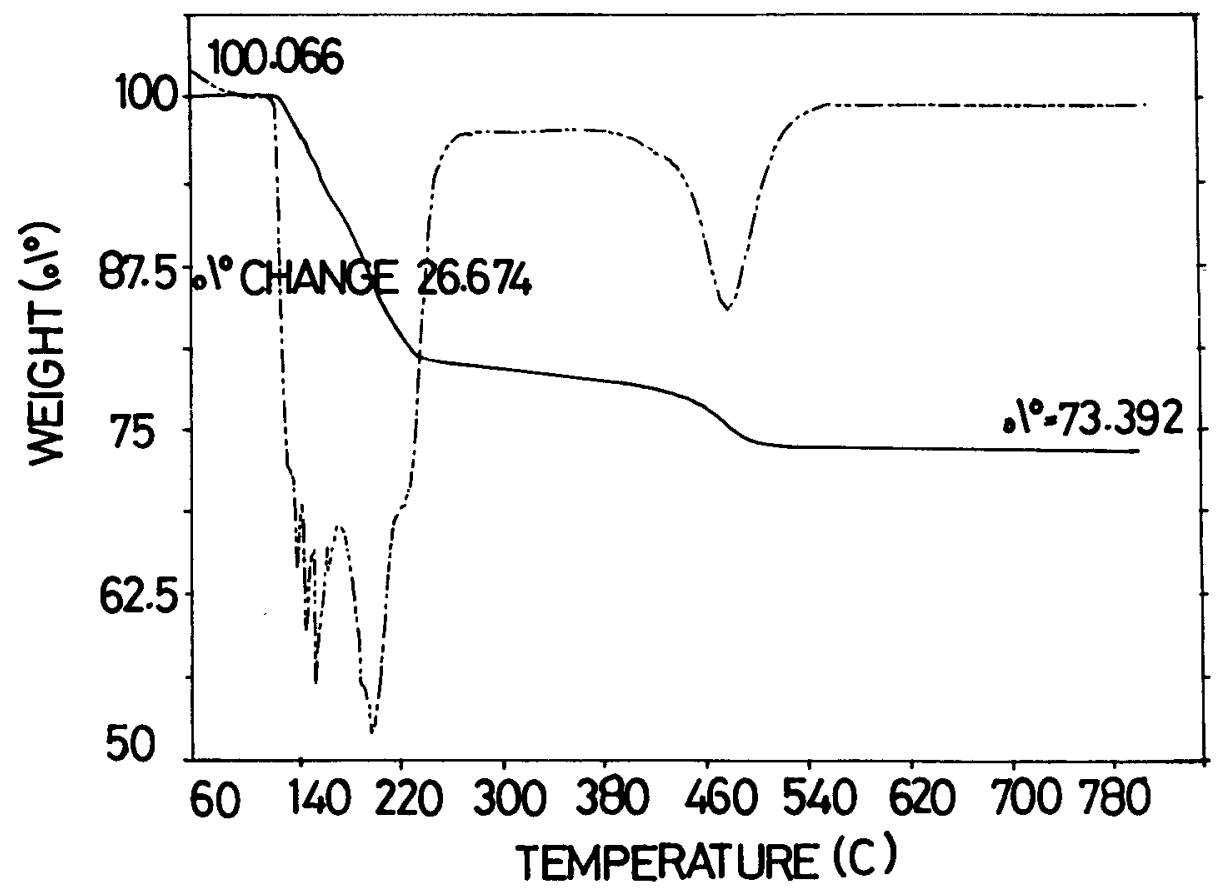

Figure 4. TG tracing of calcium pyrophosphate dihydrate in the temperature range 60 to $780^{\circ} \mathrm{C}$.

\subsection{Thermal analysis}

Thermogravimetric analysis of the crystals was carried out using a Delta series TGA 7 model recording apparatus at a heating rate of $20^{\circ} \mathrm{C} / \mathrm{min}$. Figure 4 gives the TG tracing of the crystal. Taking the initial weight as standard the course of decomposition 
is analysed by comparing their molecular weights. It is observed that calcium pyrophosphate dihydrate crystal contains two molecules of water of crystallization. Between $100^{\circ} \mathrm{C}$ and $180^{\circ} \mathrm{C}$ the TG curve shows a loss of two molecules of water of crystallization in one step. However, from the DTG curve peaks it is evident that the loss is sequential, corresponding to temperatures $160^{\circ}$ and $200^{\circ} \mathrm{C}$ respectively. In the TG curve loss seems to have taken place in one step because the $\mathrm{Ca}_{2} \mathrm{P}_{2} \mathrm{O}_{7} \cdot 2 \mathrm{H}_{2} \mathrm{O}$ crystal loses one molecule of water first. The monohydrate state has no stability region. Between $180^{\circ}$ and $460^{\circ} \mathrm{C}$ the fused calcium pyrophosphate suffers reduction to calcium phosphate.

\subsection{Optical microscopy}

Photographs of calcium pyrophosphate crystals grown by the gel technique are given in figure 5. The crystals exist in the form of rod- and needle-like crystals clustered into a spherulitic shape.

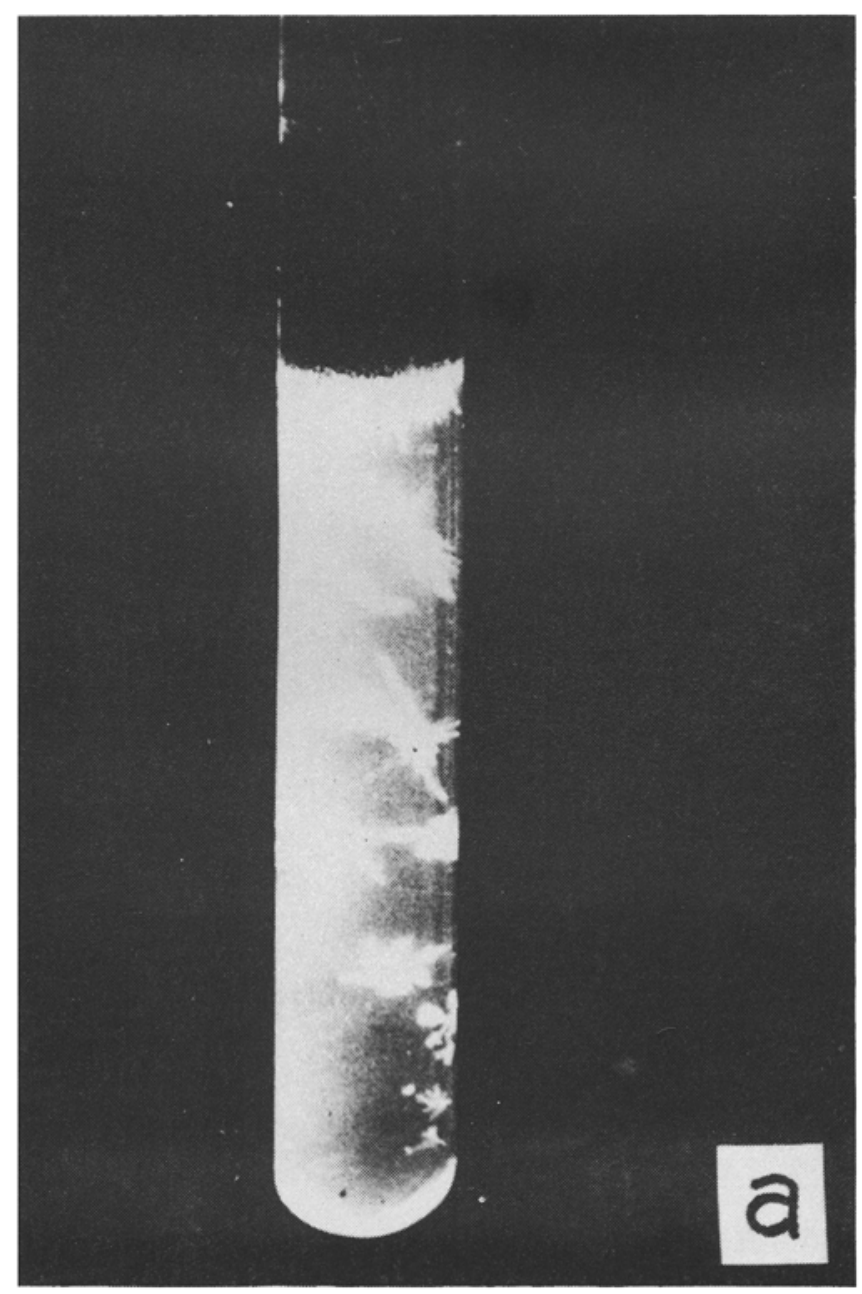

Figure 5a. For caption, see p. 110 . 


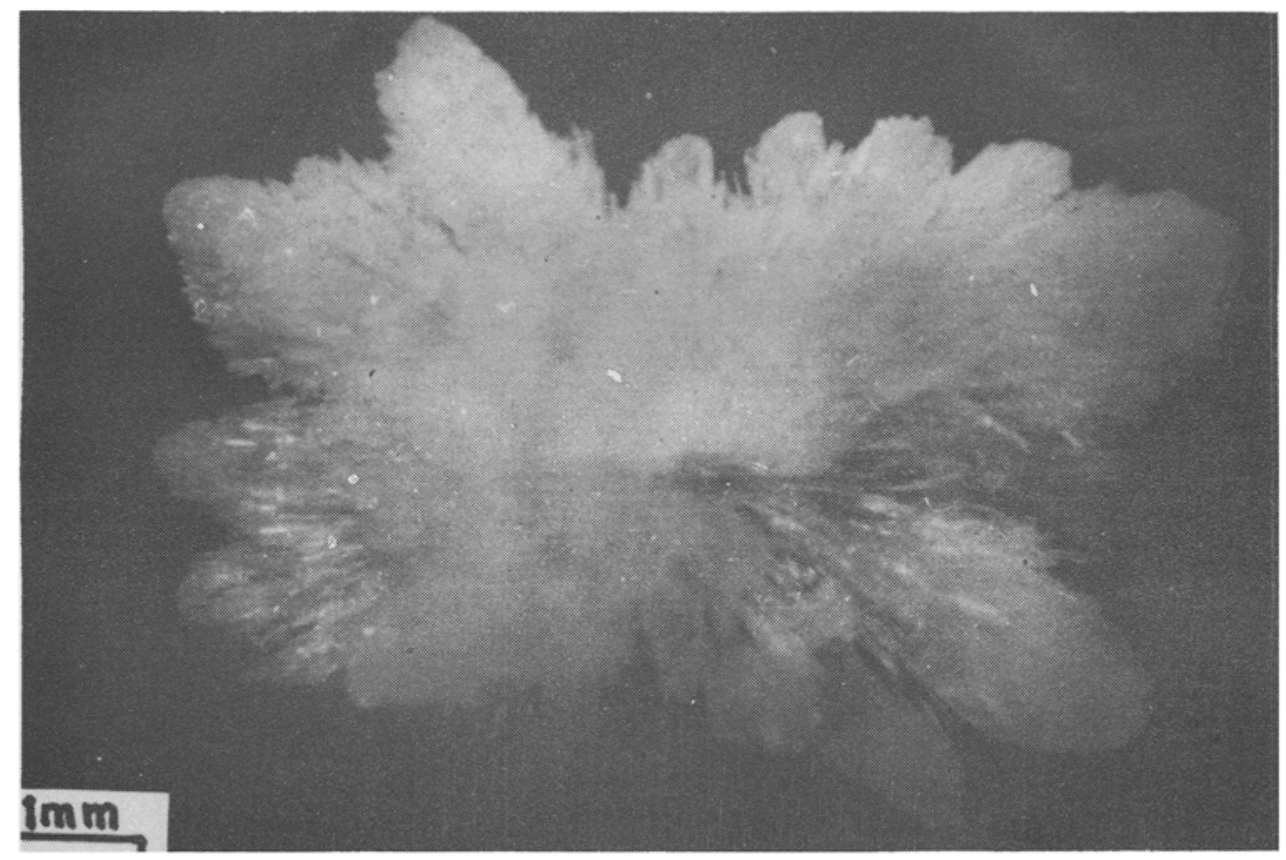

Figure 5. Optical micrographs of calcium pyrophosphate dihydrate crystals.

\section{Conclusions}

Calcium pyrophosphate dihydrate has been grown in silica gel medium. The crystal growth of calcium pyrophosphate is a very complicated process. In the present in vitro study of crystal formation, the effect of molarity of the feed solution, viz. calcium chloride, on the growth rate of the crystal is studied. From the results obtained, it is concluded that the change in the concentration of the calcium ions has a major role in the growth of the crystal.

\section{Acknowledgement}

One of the authors (MD), thanks CSIR, New Delhi, for the award of a senior research fellowship.

\section{References}

Brown E H, Lehr J R and Smith J P 1963 J. Agric. Food Chem. 11214

Corbridge D E C and Loure E J 1954 J. Chem. Soc. 76493

Law H M and Faust J W 1972 J. Cryst. Growth 13/14 471

Pritzker K P H, Cheng P T, Omar S A and Nyburg S C 1981 J. Rheumatol. 8451 